\section{Case Reports in Ophthalmology}

\title{
Genetic Background of a Recurrent Unusual Combined Form of Retinal Vein Occlusion: A Case Report
}

\author{
Kajo Bucan ${ }^{a}$ Ivna Plestina Borjan ${ }^{a}$ Ivona Bucan ${ }^{b}$ \\ Martina Paradzik Simunovic ${ }^{a}$ Ivan Borjan ${ }^{b}$ \\ aDepartment of Ophthalmology, University Hospital Split, Split, Croatia; \\ bUniversity of Split School of Medicine, Split, Croatia
}

\section{Keywords}

Plasminogen activator inhibitor-1 - PAI-1 4G/5G polymorphism - Retinal vein occlusion .

Thrombophilia

\begin{abstract}
The authors report a rare case of nonischemic branch retinal vein occlusion and nonischemic hemiretinal vein occlusion in a patient with impaired fibrinolysis. A 61-year-old woman presented to the Department of Ophthalmology, Clinical Hospital Center Split, Croatia, with acute blurring of vision in the right eye (RE) due to branch retinal vein occlusion. Ophthalmologic evaluation revealed a best corrected visual acuity (BCVA) of 0.02 in the RE and of 1.0 in the left eye. Ophthalmoscopy and fluorescein angiography of the RE demonstrated signs of nonischemic branch retinal vein occlusion. She was otherwise healthy and had no other ocular and systemic diseases. She was treated with 3 consecutive intravitreal applications of antivascular endothelial growth factor (anti-VEGF; bevacizumab) due to cystoid macular edema with full resolution of the intraretinal fluid and improvement of the BCVA to 0.9. After 8 months, she presented again with acute blurring of vision in the same (right) eye with a BCVA of 0.5. Ophthalmoscopy and fluorescein angiography of the RE indicated nonischemic hemiretinal vein occlusion. She was treated with a single intravitreal application of anti-VEGF (ranibizumab) due to macular edema. Full resolution of the intraretinal fluid and improvement of the BCVA to 0.9 were achieved. A laboratory workup was performed to rule out all known causes of retinal venous occlusive disease, which showed negative results. A molecu-
\end{abstract}




\section{Case Reports in Ophthalmology}

Case Rep Ophthalmol 2018;9:248-253

DOI: $10.1159 / 000488234$

(c) 2018 The Author(s). Published by S. Karger AG, Basel www.karger.com/cop

Bucan et al.: Genetic Background of a Recurrent Unusual Combined Form of RVO

lar analysis showed the gen of thrombophilia - plasminogen activator inhibitor (PAI)-1 4G/5G polymorphism genotype - as the only risk factor for retinal venous occlusive disease in our patient.

\section{Introduction}

Retinal venous occlusive disease is the second most common retinal vascular disorder after diabetic retinopathy. Its prevalence has been shown to vary from 0.7 to $1.6 \%[1,2]$. Depending on the location of venous blockage, retinal vein occlusion (RVO) is classified as central RVO (CRVO), branch RVO (BRVO), or hemiretinal vein occlusion (HRVO).

Early recognition and treatment are important to avoid potentially significant visual morbidity. Occlusion or thrombosis of the retinal vein is associated with chronic glaucoma, atherosclerotic risk factors (age, diabetes, and hypertension), hyperviscosity, coagulopathy, smoking, and migraine [3].

Many authors consider different pathogenetic mechanisms in most cases of CRVO and BRVO. CRVO can occur as a result of various pathologic insults, including compression of the vein (mechanical pressure due to arteriosclerotic changes in the central retinal artery and/or structural changes in the lamina cribrosa), hemodynamic disturbances (associated with hyperdynamic or sluggish circulation), vasculitis (e.g., sarcoidosis), and changes in the coagulating factors in the blood (e.g., deficiency of thrombolytic factors, increase in clotting factors). Hemicentral or hemispheric RVO, a variant of CRVO, is associated with congenital variation in central vein anatomy, and it may involve either the superior or the inferior half of the retina.

An idiopathic thrombus at an arteriovenous crossing site typifies the presentation of nearly all BRVOs. At arteriovenous crossing sites, the artery and the vein have a common adventitial sheath. Like systemic vascular disease, hypertrophy of the arterial wall media results in artery enlargement and rigidity. The venous lumen is thereby compressed and narrowed, causing endothelial cell damage and turbulent blood flow. Thrombus formation via platelet adherence and aggregation results in clinically evident BRVO [4-6].

We present a case of recurrent nonischemic combined RVO (branch and hemicentral) in a patient with impaired fibrinolysis.

\section{Case Report}

A 61-year-old woman presented to our department with sudden vision loss in her right eye (RE). Visual acuity (VA) was 0.02 in her RE and 0.9 in her left eye. Intraocular pressure and the anterior ocular segment were normal in both eyes. Ophthalmoscopic examination of the left eye was normal, but the RE revealed retinal flame-shaped hemorrhage and cotton wool spots (Fig. 1a). The early phase of fluorescein angiography showed multiple areas of hypofluorescence due to intraretinal hemorrhages and leakage of dye involving the fovea in the late phase (Fig. 1b, c). Optical coherence tomography (OCT) showed an accumulation of intraretinal fluid (Fig. 2a). The patient was otherwise healthy and had no other ocular or systemic diseases. The results of electrocardiography and laboratory tests (full blood count and erythrocyte sedimentation rate or plasma viscosity, urea, electrolytes, creatinine, random blood glucose, random cholesterol and HDL cholesterol, plasma protein electrophoresis, and thyroid function), including blood coagulation and the immune system, were normal. 
She was treated with 3 consecutive intravitreal applications of anti-vascular endothelial growth factor (anti-VEGF; bevacizumab) due to cystoid macular edema, with improvement of the best corrected VA (BCVA) to 0.9 and full resolution of the intraretinal fluid on OCT (Fig. 2b).

After 8 months, she presented again to our department with acute blurring of vision in the same (right) eye with a BCVA of 0.5. An ophthalmoscopic examination of the RE revealed retinal flame-shaped hemorrhages and cotton wool spots, but this time in a completely inferior part of the retina. Fluorescein angiography of the RE showed signs of nonischemic HRVO (Fig. 3a, b). She was treated with a single intravitreal application of anti-VEGF (ranibizumab) due to perifoveal edema as seen on OCT, with resolution of the intraretinal fluid and improvement of the BCVA to 0.9 (Fig. 3c, d).

Further laboratory tests including prothrombin time, activated partial thromboplastin time, protein $\mathrm{C}$, protein S, factor V Leiden, antithrombin III, homocysteine, antinuclear antibody, lupus anticoagulant, and anticardiolipin antibody were performed and showed negative results.

A molecular analysis was also performed. The patient was tested for five gene variants: factor V Leiden (FVQ506), factor II (G20210A), 5,10-methylenetetrahydrofolate reductase (MTHFR) (C677T), plasminogen activator inhibitor-1 (PAI-1) (4G/4G, 5G/5G, and 4G/5G), and angiotensin-converting enzyme (del/ins). By amplifying the promoter region of the gene for PAI-1, the insertion-deletion genotype $(4 \mathrm{G} / 5 \mathrm{G})$ was determined in the patient. Other tests showed a normal type of the tested genes.

\section{Discussion}

RVO is a disease of the older population (aged $>65$ years). The major risk factors are hypertension, diabetes, and atherosclerosis. Other risk factors are glaucoma, syphilis, sarcoidosis, vasculitis, anemia, increased orbital or intraocular pressure, hyperviscosity syndromes (multiple myeloma, Waldenström macroglobulinemia, and leukemia), high homocysteine levels, sickle cells, and HIV [7]. In addition to ruling out known causes of vasculitis, such as Behçet disease, sarcoidosis, syphilis, and Lyme disease, tests to consider include levels of serum homocysteine, antinuclear antibody, lupus anticoagulant, and anticardiolipin antibody. In our case, no abnormality was observed.

PAI-1 is a fast-acting inhibitor of tissue-type plasminogen activator. Marcucci et al. [8] found elevated levels of PAI-1 in patients with CRVO compared with those in control subjects. PAI-1, also known as endothelial plasminogen activator inhibitor or serpin E1, is a protein encoded by the serpine 1 gene in humans. The main function of PAI-1 entails the inhibition of urokinase plasminogen activator, an enzyme responsible for the cleavage of plasminogen to form plasmin. Elevated PAI is a risk factor for thrombosis and atherosclerosis [9]. Presently, routine testing of patients for PAI-1 is not recommended. Laboratory evaluations are not recommended in typical BRVO, while atypical cases such as BRVO occurring in young patients, bilateral BRVO, or BRVO occurring at a site not associated with an arteriovenous crossing warrant a laboratory workup.

The PAI-1 gene is serpine 1, located on chromosome 7 (7q21.3-q22). There is a common polymorphism, known as $4 \mathrm{G} / 5 \mathrm{G}$, in the promoter region. The $4 \mathrm{G}$ allele frequency of the PAI1 gene, associated with hypofibrinolysis, was found to be $64 \%$ in cases of thrombophilia in the study by Glueck et al. [10], and much less in control cases. The PAI-1 gene 4G allele was associated with RVO with an odds ratio of 1.94 . Increased PAI-1 activity in patients with RVO 
was determined also by PAI-1 4G/5G polymorphisms by Gori et al. [11]. PAI-1 activity was determined by the PAI-1 4G/5G polymorphism by polymerase chain reaction and restriction length fragment polymorphism. In another study, Mattes et al. [12] found that among all patients with RVO, 54.5\% had the PAI-1 4G/5G gene polymorphism. Conversely, for the angiotensin-converting enzyme (ins/del) and PAI-1 (4G/5G) mutations, no difference was observed between the genotypes of patients with RVO and those of control participants in the study by Russo et al. [13] and Kuhli-Hattenbach et al. [14]. Homozygosity for the PAI-1 4G/5G gene polymorphism itself was not associated with an increased risk for RVO, with no significant difference in PAI-1 4G/5G genotype distribution with either retinal artery or vein occlusion. However, previous studies have shown no clinical appearance of unilateral recurrent nonischemic BRVO and HRVO in a patient with no systemic associations and a normal laboratory workup for thrombophilia [15].

\section{Conclusion}

Our case illustrates a unique presentation of unilateral recurrent nonischemic BRVO and HRVO in the same eye in a patient with no systemic associations and a normal laboratory workup for thrombophilia. A molecular analysis of the genes associated with thrombophilia showed that the PAI-1 4G/5G polymorphism genotype could be responsible for the retinal venous occlusive disease in our patient. Few and conflicting data are available for this genotype of thrombophilia associated with RVO. Our case demonstrated genetically impaired fibrinolysis as the only risk factor for this condition.

\section{Statement of Ethics}

The authors have no ethical conflicts to disclose.

\section{Disclosure Statement}

The authors declare that they have no competing interests.

\section{Funding Sources}

There was only institutional support from the University Hospital Split. There were no other funding sources.

\section{Author Contributions}

The case report was written by all authors. All authors read and approved the final manuscript. 


\section{Case Reports in Ophthalmology}

\section{References}

1 Klein R, Klein BE, Moss SE, Meuer SM. The epidemiology of retinal vein occlusion: the Beaver Dam Eye Study. Trans Am Ophthalmol Soc. 2000;98:133-41; discussion 141-3.

2 Mitchell P, Smith W, Chang A. Prevalence and associations of retinal vein occlusion in Australia. The Blue Mountains Eye Study. Arch Ophthalmol. 1996 Oct;114(10):1243-7.

3 Klein R, Moss SE, Meuer SM, Klein BE. The 15-year cumulative incidence of retinal vein occlusion: the Beaver Dam Eye Study. Arch Ophthalmol. 2008 Apr;126(4):513-8.

4 Prisco D, Marcucci R. Retinal vein thrombosis: risk factors, pathogenesis and therapeutic approach. Pathophysiol Haemost Thromb. 2002 Sep-Dec;32(5-6):308-11.

5 Cugati S, Wang JJ, Knudtson MD, Rochtchina E, Klein R, Klein BE et al. Retinal vein occlusion and vascular mortality: pooled data analysis of 2 population-based cohorts. Ophthalmology. 2007 Mar;114(3):520-4.

6 Biousse V, Newman NJ, Sternberg P Jr. Retinal vein occlusion and transient monocular visual loss associated with hyperhomocystinemia. Am J Ophthalmol. 1997 Aug;124(2):257-60.

7 Bhagat N, Goldberg MF, Gascon P, Bell W, Haberman J, Zarbin MA. Central retinal vein occlusion: review of management. Eur J Ophthalmol. 1999 Jul-Sep;9(3):165-80.

8 Marcucci R, Bertini L, Giusti B, Brunelli T, Fedi S, Cellai AP et al. Thrombophilic risk factors in patients with central retinal vein occlusion. Thromb Haemost. 2001 Sep;86(3):772-6.

9 Vaughan DE. PAI-1 and atherothrombosis. J Thromb Haemost. 2005 Aug;3(8):1879-83.

10 Glueck CJ, Wang P, Bell H, Rangaraj V, Goldenberg N. Associations of thrombophilia, hypofibrinolysis, and retinal vein occlusion. Clin Appl Thromb Hemost. 2005 Oct;11(4):375-89.

11 Gori AM, Marcucci R, Fatini C, Gensini F, Sticchi E, Sodi A et al. Impaired fibrinolysis in retinal vein occlusion: a role for genetic determinants of PAI-1 levels. Thromb Haemost. 2004 Jul;92(1):54-60.

12 Mattes D, Weger M, Renner W, Cichocki L, Haas A. Role of angiotensin-converting enzyme insertion/deletion and plasminogen activator inhibitor-14G/5G gene polymorphisms in retinal vein occlusion. J Thromb Haemost. 2005 Nov;3(11):2583-4.

13 Russo PD, Damante G, Pasca S, Turello M, Barillari G. Thrombophilic mutations as risk factor for retinal vein occlusion: a case-control study. Clin Appl Thromb Hemost. 2015 May;21(4):373-7.

14 Kuhli-Hattenbach C, Hellstern P, Nägler DK, Kohnen T, Hattenbach LO. Prothrombin polymorphism A19911G, factor V HR2 haplotype A4070G, and plasminogen activator-inhibitor-1 polymorphism 4G/5G and the risk of retinal vein occlusion. Ophthalmic Genet. 2017 Sep-Oct;38(5):413-7.

15 Balta G, Altay C, Gurgey A. PAI-1 gene 4G/5G genotype: a risk factor for thrombosis in vessels of internal organs. Am J Hematol. 2002 Oct;71(2):89-93.
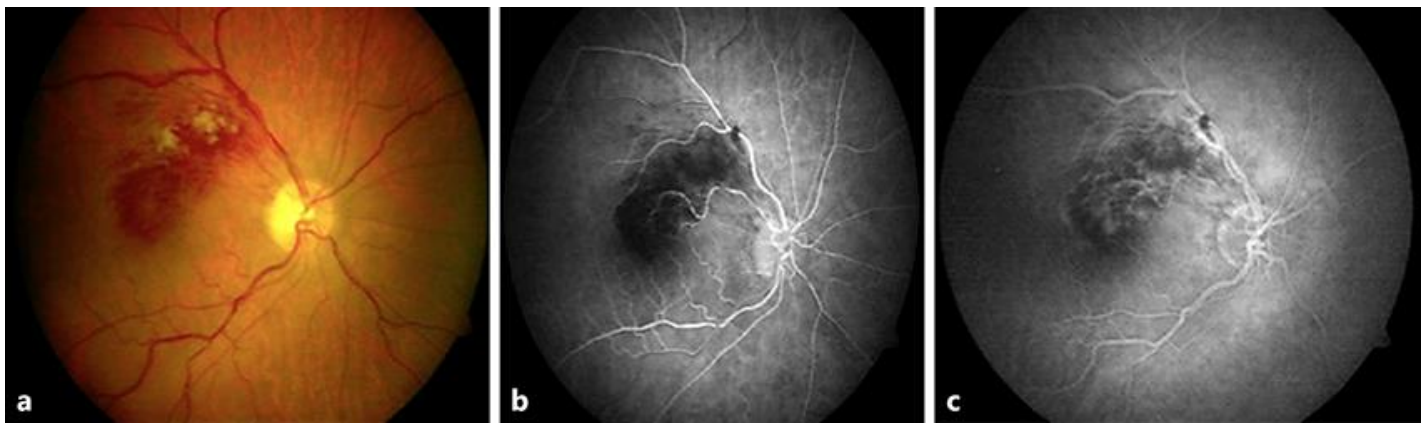

Fig. 1. a Color fundus photograph of the right eye showing upper branch retinal vein occlusion with cotton wool spots and flame-shaped hemorrhages in the areas drained by the affected vein. b, c Fluorescein angiograms showing areas of hypofluorescence due to intraretinal hemorrhages in the early phase and leakage of dye involving the fovea in the late phase. 


\section{Case Reports in Ophthalmology}
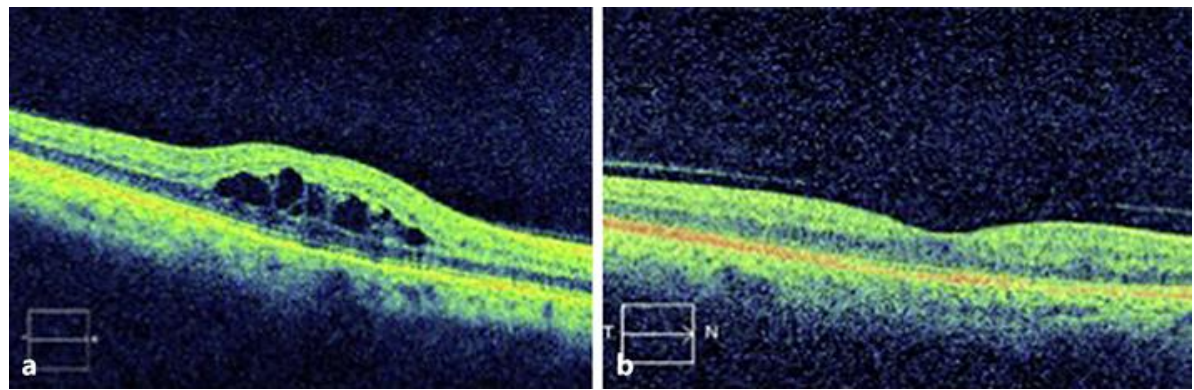

Fig. 2. a OCT image demonstrating a cystoid macular edema. b OCT image after 3 consecutive applications of intravitreal bevacizumab demonstrating full resolution of the intraretinal fluid and a preserved foveal contour.
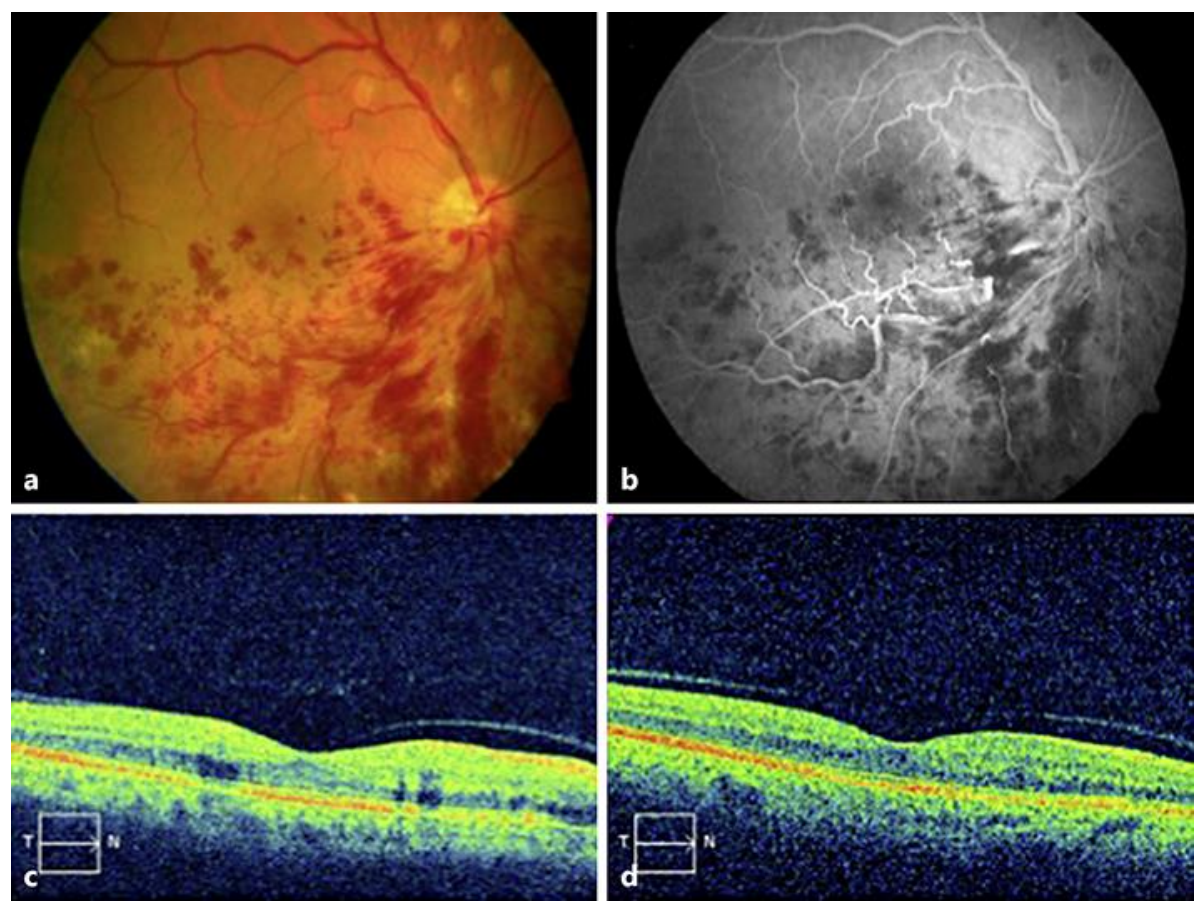

Fig. 3. a Color fundus photograph of the right eye showing hemiretinal vein occlusion with flame-shaped and dot hemorrhages in areas drained by the affected vein. b Fluorescein angiogram showing multiple areas of hypofluorescence due to intraretinal hemorrhages and subtle leakage of dye in the foveal area, with no signs of perfusion. c OCT image demonstrating a subtle perifoveal edema. $\mathbf{d}$ OCT image after a single application of intravitreal ranibizumab, demonstrating resolution of the perifoveal fluid. 